\title{
Aves del sendero Cóndor Machay (cantón Rumiñahui, Pichincha- Ecuador): Evaluación de especies con valor para el aviturismo local
}

\section{Birds of the Cóndor Machay trail (cantón Rumiñahui, Pichincha- Ecuador): Evaluation of species with local avitouristic value}

\author{
Iván Vinicio Jácome-Negrete ${ }^{1}$, Henry Paúl Monar-Barragán²* \\ ${ }^{1}$ Facultad de Ciencias Biológicas de la Universidad Central del Ecuador, calle Iquique N 14-121 y Sodiro-Itchimbía, \\ EC170136, Quito, Ecuador. \\ https://orcid.org/0000-0002-8337-7959 \\ ${ }^{2}$ Facultad de Ciencias Biológicas de la Universidad Central del Ecuador, calle Iquique N 14-121 y Sodiro-Itchimbía, \\ EC170136, Quito, Ecuador. \\ https://orcid.org/0000-0002-9079-0012
}

凶 Autor para correspondencia: hpmonar@uce.edu.ec

\section{Resumen}

Se planteó este estudio con el objetivo de evaluar el potencial aviturístico de las aves del sendero Cóndor Machay, localizado en el bosque montano alto, de la parroquia Rumipamba, en el cantón Rumiñahui (provincia de Pichincha-Ecuador). Las aves fueron identificadas visualmente mediante once recorridos de 3,5 km efectuados desde el sector de La Caldera a la cascada Cóndor Machay, entre septiembre de 2017 y marzo de 2018. Para cada especie se calculó su valor aviturístico, como una variable que combina los parámetros de estética, abundancia relativa, perceptibilidad, estado de conservación, endemismo, importancia científica, valor histórico cultural y singularidad taxonómica. En total, se registraron 58 especies de 21 familias y 9 órdenes, con predominio de colibríes (Trochilidae) y Passeriformes. Las diez especies con mayor valor aviturístico fueron: caracara curiquingue, tangara montana ventriescarlata, gaviota andina, mirlo grande, carpintero dorsicarmesí, matorralero nuquirufo, colibrí orejivioleta ventriazul, candelita de anteojos, gallinazo negro y la pava andina. El sendero constituye un refugio para las aves andinas locales y presenta un alto valor para el fomento del aviturismo local. Se recomienda asegurar su conservación a largo plazo, a través de la regulación de la capacidad de carga turística y la aplicación estricta de normas para el visitante, en cuanto a la disposición final de la basura e introducción de mascotas dentro del sendero.

Palabras clave: Andes, aves, bosque montano, diversidad, Ecuador.

\begin{abstract}
This study was designed with to evaluate the bird watching potential in the Cóndor Machay trail. This trail is located in the high montane forest, of Rumipamba parish, in the Rumiñahui canton (Pichincha Province-Ecuador). The birds were visually identified by eleven walk-rounds of $3.5 \mathrm{~km}$ carried out from La Caldera sector to the Cóndor Machay waterfall, from September 2017 to March 2018. For each species, its bird watching values were estimated as a variable that combines the parameters of aesthetics, relative abundance, perceptibility, conservation status, endemism, value, and scientific importance, cultural historical value and taxonomic singularity. In total, 58 species of 21 families and 9 orders were registered, with a predominance of hummingbirds (Trochilidae) and Passeriformes. 10 species with the greatest avituristic value were: Carunculated Caracara, Scarlet-bellied Mountain-Tanager, Andean Gull, Great Thrush, Crimson-mantled Woodpecker, Yellow-breasted Brush-Finch, Sparkling Violetear, Spectacled Redstart, American Black Vulture and the Andean Guan. The trail is a refuge for
\end{abstract}


local Andean birds, and has a high value for the promotion of the local bird-watching tourism activities. To ensure the long-term conservation of this site, we recommend regulating the tourist carrying capacity and to implement strict rules for the visitors regarding garbage disposal and pet handling within the path.

Keywords: Andes, birds, montane forest, diversity, Ecuador.

\section{Introducción}

A escala mundial existen 34 hotspots; de éstos, 25 se localizan parcial o totalmente en las montañas (Price et al., 2011). La cordillera de los Andes tropicales presenta una alta diversidad ecosistémica y biológica debido a la existencia de gradientes geográficos y ambientales (Josse et al., 2009). Toda esta región se caracteriza por su alto endemismo, los múltiples servicios ecosistémicos que provee en términos de regulación climática y captura de carbono (Tejedor et al., 2012), y por ser parte del patrimonio forestal de los países andinos. Los bosques montanos registran cifras de alta diversidad vegetal al norte de los Andes. En Ecuador se localizan por encima de los $3.000 \mathrm{~m}$ y constituyen la vegetación de transición, localmente conocida como ceja andina, la cual se ubica entre los bosques nublados y el páramo (Cuesta et al., 2009; MAE \& FAO, 2015).

Los bosques montanos andinos registran un alto endemismo, especialmente de plantas y vertebrados (Price et al., 2011). En Ecuador, el bosque montano contiene el $50 \%$ de todas las especies de la flora ecuatoriana (MAE \& FAO, 2015). Estos ecosistemas brindan múltiples servicios ecosistémicos, como la oferta de productos forestales, protección de laderas, retención de agua, además de ser espacios importantes para la supervivencia de poblaciones humanas (Cuesta et al., 2009; Hostettler, 2002; Price et al., 2011). Si bien a escala regional, la cobertura natural andina aún cubre un $79 \%$ (Josse et al., 2009), la situación de Ecuador difiere, la remanencia de los bosques montanos únicamente alcanza un 11 \% (Cuesta et al., 2009). La principal causa de conversión de la cobertura natural de los bosques montanos es la implantación de tierras agrícolas (Price et al., 2011), seguido por las concesiones mineras y la presión por el fuego. La quema y tala de bosques montanos, realizadas por agricultores andinos, han provocado la pérdida de un $45 \%$ de los bosques originales desde 1980 (MAE \& FAO, 2015).

Dentro del cantón Rumiñahui quedan pocos remanentes de bosque montano andino, ubicados en las faldas del volcán Pasochoa, sobre los 3.200 m s.n.m.
La vegetación natural ha quedado reducida al $10 \%$ de su cobertura original por factores como la agricultura intensiva, ganadería, cultivo de bosques de eucalipto y el avance de la expansión urbana e industrial (Sociedad Civil del cantón Rumiñahui, 2001).

Para la zona andina, Fjeldså (2007) recomienda que los esfuerzos tradicionales para preservar la biodiversidad se complementen con actividades que promuevan el desarrollo sostenible en las áreas pobladas. Desde esta perspectiva y considerando su alto valor paisajístico, estos bosques pueden ser la base para el fomento del turismo (Price et al., 2011), que permita su conservación a largo plazo. El aviturismo es una de las pocas actividades que cubren las expectativas de los conceptos de ecoturismo y sustentabilidad, al ofrecer a los interesados el contacto directo con la naturaleza (Amparán \& Téllez, 2005). En este contexto, esta actividad es una opción económica viable para la conservación del patrimonio biocultural (CAF-Banco de Desarrollo de América Latina, 2014). El aviturismo como actividad recreativa, además de descubrir la riqueza de las aves y favorecer su conservación, también contribuye a mejorar las condiciones de vida de las personas con la creación de festivales turísticos como el Festival de las Aves de las Altas Montañas de Veracruz, en México (Pérez-Sato et al., 2018). Otro ejemplo es la realización de los "Birding Rally Challenges", como el realizado entre equipos internacionales de observadores apasionados de las aves que cubrieron una ruta montañosa entre Lima y Cusco, en Perú, durante 5 días de competencia, con el triunfo de un equipo estadounidense que logró registrar 493 especies de aves (Verástegui, 2013).

En Ecuador, la ornitofilia, entendida como la afición hacia las aves, comenzó a partir de 1980, y en la actualidad es una actividad económica de importancia dentro del turismo de naturaleza (Freile \& Rodas, 2008). El turismo relacionado con la observación de aves es un producto tradicional con alto potencial de exportación (Mindo Cloudforest Foundation, 2006). En el país, la práctica del aviturismo, además de ser una actividad eco- 
nómica generadora de fuentes de trabajo, también contribuye a la protección del ambiente (Herrera \& Lasso, 2014). El aviturismo también ha favorecido el desarrollo local al proponer alternativas sostenibles que vinculan la conservación de las aves con la generación de empleo para personas de comunidades rurales, como ha ocurrido con la creación de rutas aviturísticas en comunidades como las cuatro rutas de aves de Gualchán, en Carchi (Chandi \& Muñoz, 2018). El país se ha posicionado como un destino aviturístico de notable importancia a nivel regional y mundial (Steven, 2017). Sin embargo, la mayor afluencia de turistas en áreas protegidas también puede ser un factor de introducción de especies exóticas, lo cual implica un riesgo para la conservación (Muñoz, 2015).

El aviturismo, como componente del ecoturismo, se enfoca en la observación de las aves, y si se lo realiza sustentablemente genera beneficios económicos (dinero a partir de la oferta de servicios de transporte, hospedaje, comida, venta de recuerdos, generación de empleo), sociales (promoción del desarrollo rural) y de conservación (protección local de las especies) (El-Mahdy \& Abdel-Motaal, 2018). El aviturismo, como producto turístico, implica el desarrollo de varias actividades: un diagnóstico de la riqueza de las aves del sitio, el diseño de posibles paquetes y rutas aviturísticas, un detallado análisis de costos, la definición de estrategias de mercado y comercialización, un análisis de la viabilidad legal del proyecto y un estudio económico y financiero de la rentabilidad del mismo (Llulluna, 2018).

El diagnóstico de la biodiversidad local es un elemento clave para el desarrollo del aviturismo. Los estudios sobre la avifauna del cantón Rumiñahui son escasos. El Gobierno Autónomo Descentralizado de Rumiñahui (2011) publicó un atlas de la biodiversidad del cantón, en el cual se repor$\tan 61$ especies de aves, de las cuales, 52 son propias del bosque montano, con predominio de Passeriformes y Apodiformes. Se menciona también la existencia de tres especies endémicas de laderas y valles interandinos, una especie amenazada y catorce especies más registradas en el Apéndice II de CITES. Guarderas \& Jácome (2010) publican una guía de aves del cantón para la zona urbana y algunos sectores rurales en la que se reporta la existencia de 40 especies más comunes. Se menciona que a medida que se profundicen los estudios esta cifra inicial podría incrementarse, tomando en cuenta la ubicación de las áreas rurales en las faldas del volcán Pasochoa. Se indica que debido a la creciente deforestación y urbanización del cantón solamente quedan relictos de bosque montano en los sitios más inaccesibles, y que las áreas verdes ofrecen condiciones adecuadas para la existencia de la avifauna local al no contar con cobertura arbustiva natural. Según el Plan de desarrollo y ordenamiento territorial de la parroquia Rumipamba, 2012-2025 (Gobierno Autónomo Descentralizado Parroquial de Rumipamba, 2012), se indica que a pesar de existir zonas de alto valor paisajístico y multiplicidad de pisos, esta parroquia registra los valores más altos en los indicadores de pobreza, con insuficiencia de fuentes de trabajo, al estar centradas las actividades productivas de agricultura y ganadería, sin incursionar en otras actividades compatibles con la conservación.

En la última década se ha promocionado el desarrollo de nuevos espacios como áreas para la recreación y el turismo. Uno de estos espacios es el sendero que conduce a la cascada Cóndor Machay. Este sendero atraviesa uno de los últimos remanentes de bosque montano alto a lo largo del río Pita, y tiene una extensión aproximada de $3,5 \mathrm{~km}$. Sin embargo, no existen estudios actualizados que permitan conocer una cifra real de las especies de aves que hay en la zona y tampoco se ha realizado una valoración del potencial aviturístico que pueden tener las especies. Tomando en cuenta lo mencionado, el presente estudio desarrolló una evaluación del potencial aviturístico de las aves que habitan el sendero peatonal. Se espera contribuir, a través de este trabajo, con la promoción del aviturismo local como una estrategia que dinamice la economía y, a la vez, contribuya a la conservación de esta área.

\section{Materiales y métodos}

\section{1. Área de estudio}

El área de estudio se localiza en la provincia de Pichincha, cantón Rumiñahui, parroquia Rumipamba, a una altura referencial de 3.100 m s.n.m. El sitio específico se localizó en el sendero turístico de acceso a la cascada Cóndor Machay, desde el sitio identificado como La Caldera $\left(0,456491^{\circ} \mathrm{S} ; 78,423584^{\circ} \mathrm{O}\right.$ Datum WGS84) hasta la cascada $\left(0,482283^{\circ} \mathrm{S}\right.$; $78,429047^{\circ} \mathrm{O}$ Datum WGS84), con una longitud de $3,5 \mathrm{~km}$, a las orillas del río Pita (Figura 1). 


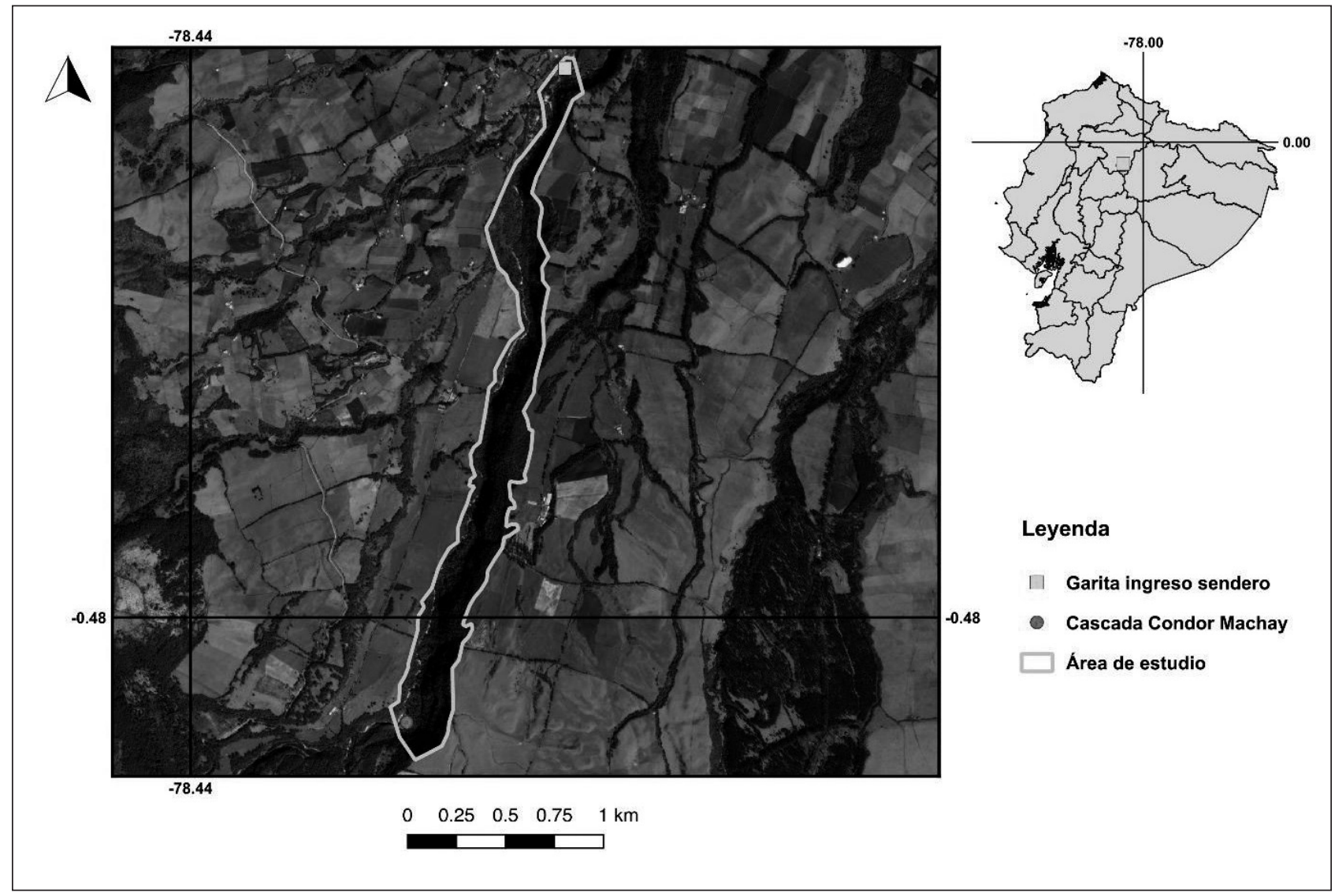

Figura 1. Mapa del área de estudio (Fuente: Google Earth, 2017).

El sendero está rodeado por un relicto de bosque montano alto, identificado conforme la clasificación propuesta por MAE \& FAO (2015). Predominan especies arbóreas de las familias Araliaceae, Betulaceae, Buddlejaceae, Cunnoniaceae, Euphorbiaceae, y arbustivas de las familias Asteraceae, Boraginaceae, Campanulaceae, Caprifoliaceae, Clusiaceae. En cuanto a su zoogeografía, la zona de estudio pertenece al piso altoandino, de acuerdo con la clasificación propuesta por Albuja et al. (2012).

El sendero como recurso turístico está bajo la administración de la Dirección de Turismo del GAD Rumiñahui, desde el 2007 (Herrera, 2016). Con aporte municipal se han construido puentes de acceso, señalética, una garita y se cuenta con la presencia permanente de un guardaparque que controla las actividades turísticas.

\subsection{Metodología}

Para la evaluación del potencial de las aves del sendero Cóndor Machay, desde una perspectiva avi- turística, se aplicó la secuencia metodológica de los apartados 2.2.1. y 2.2.2.

\subsubsection{Inventario de especies}

Se realizó un inventario ornitológico con énfasis en las aves detectables a simple vista del área. El registro de las aves observables se hizo mediante el establecimiento de un transecto de 3,5 $\mathrm{km}$ de distancia, sobre el sendero de acceso a la cascada, desde la garita de ingreso al sitio en $\mathrm{La}$ Caldera hasta la cascada Cóndor Machay.

El sendero fue recorrido a partir de las 07:00 hasta las 13:00, de ida y vuelta a la cascada. Se realizaron 11 recorridos, desde el 01 de septiembre de 2017 hasta el 22 de marzo de 2018, con dos recorridos mensuales. El sendero fue dividido en 11 sectores de registro, aprovechando la existencia de puentes metálicos $(\mathrm{P})$ como límites entre sectores. Se dividió de la siguiente manera: Sector $\mathrm{S} 0=$ garita a $\mathrm{p} 1$, Sector $12=$ puente 1 a $\mathrm{p} 2$, Sector $23=\mathrm{p} 2$ a p3, Sector $34=\mathrm{p} 3$ a p4, Sector $45=\mathrm{p} 4$ 
a p5, Sector $56=\mathrm{p} 5$ a p6, Sector $67=\mathrm{p} 6$ a p7, Sector $78=$ p7 a p8, Sector $89=$ p8 a p9, Sector $90=$ p9 a p10 y Sector $100=$ p10 a cascada Cóndor Machay.

Durante cada recorrido se hicieron registros visuales de las aves, empleando monoculares (spotting scope) Bushnell (20 x 40-60) y BAK4/ FMC 12 x 50. Para cada observación realizada se registró la identidad taxonómica de cada ave, con apoyo de las guías de campo: McMullan \& Vásquez (2009) y McMullan \& Navarrete (2017). Además, se consideraron los datos: número de individuos, distancia perpendicular en metros, sector de avistamiento, hábitat, estrato, sustrato y estructura social; conforme recomiendan Villarreal et al. (2004).

Para estimar la distancia de las observaciones se empleó un medidor digital laser Bushnell Scout 1000 ARC DX. Al final de la fase de campo se elaboró una tabla con las especies registradas y sus frecuencias de avistamientos parciales y totales.

Para evaluar el esfuerzo de muestreo realizado se proyectó la curva de acumulación de especies, usando el software StimateS (Colwell, 2013), y se calculó el estimador Chao1 para cuantificar la riqueza esperada, además del porcentaje de completitud alcanzado en el muestreo, conforme recomiendan Villarreal et al. (2004). La estructura de la comunidad de aves se proyectó en una curva de rango-abundancia, cuyo eje $X$ corresponde a las especies organizadas y el eje $Y$ corresponde al logaritmo base 10 de la proporción de la abundancia relativa de cada especie respecto a la abundancia total, de acuerdo con Feinsinger (2003).

\subsubsection{Valor aviturístico potencial de las especies registradas (PAV)}

El valor aviturístico potencial de cada especie registrada en el sendero fue tabulado tomando en consideración los siguientes parámetros: Valor estético (Ve), Abundancia relativa (Ab), Perceptibilidad del ave $(\mathrm{Pe})$, Estado de conservación (Ec), Endemismo (End), Valor de uso e importancia científica $(\mathrm{Vu})$, Valor histórico cultural $(\mathrm{Vh})$ y Singularidad taxonómica (St). Cada parámetro tiene a su vez subcategorías de calificación, de acuerdo a criterios sugeridos por Muñoz-Pedreros \& Quintana (2010) y Almendras, Ferrari \& Diez (2017). Se realizaron ajustes en concordancia con las características de la avifauna bajo estudio, que se detallan en la descripción de los correspondientes subparámetros.

El potencial aviturístico de cada especie (PAV) resulta de la sumatoria de los valores de los parámetros antes indicados, empleando la fórmula [1]. La sumatoria máxima para la variable PAV es de 53,25 puntos.

\section{$P A V=\sum 1,5 \mathrm{Ve}+1,5 \mathrm{Ab}+1,5 \mathrm{Pe}+\mathrm{Ec}+\mathrm{End}+0,5 \mathrm{Vu}+0,5 \mathrm{Vh}+0,5 \mathrm{St}$}

A continuación, se detallan los criterios empleados para cada parámetro:

- Valor estético (Ve): valoración cuantitativa de la percepción estética realizada por un grupo de 25 personas, sobre los componentes visuales (colores, formas) que conforman las imágenes de la fauna presentadas a los evaluadores, siguiendo lo sugerido por Muñoz-Pedreros (2004) con modificaciones a la escala de valoración. Se mostraron imágenes a color, en su silueta lateral y de alta resolución de las 58 especies de aves registradas con una caden- cia de 20 segundos. Se consideró una escala de 0 a 3 , siendo 0 el puntaje para lo menos atractivo visualmente hasta 3 como el puntaje considerado para lo más atractivo. Cada persona dio una calificación por especie. Luego los puntajes individuales fueron promediados para obtener un puntaje final por especie.

- Abundancia (Ab): número relativo de individuos de cada especie registrados en el área de estudio. Como criterios de valoración se consideró lo propuesto por Cadena-Ortiz et al. (2015) (Tabla 1). 
Tabla 1. Criterios de valoración y puntajes para la variable Abundancia

\section{Criterio de valoración}

Puntaje

(V) registro ocasional o posible especie vagabunda

$\mathrm{R}=$ raro, registrado pocos individuos y no diariamente

$\mathrm{PC}=$ poco común, registrado diariamente en números pequeños, 1-10 individuos/día

- $\quad$ Perceptibilidad (Pe): variable que contiene los subparámetros de medición de la fórmula [2], y su correspondiente métrica. La sumatoria máxima de los subparámetros de la variable
Perceptibilidad es de 22,5 puntos. Esta sumatoria a su vez se multiplica por un coeficiente de 1,5 pudiendo alcanzar un valor máximo de 33,75 puntos.

$$
P e=1,5 \mathrm{~Pa}+1,5 \mathrm{Cm}+1,5 \mathrm{~T}+\mathrm{Pa}+\mathrm{C}+0,5 \mathrm{Pi}+0,5 \mathrm{Gt}
$$

- Periodo de actividad (Pa): período del día en el cual las especies realizan sus actividades, excluyendo sus periodos de sueño o aletar- gamiento y que guarda concordancia con la mayor factibilidad de observación de las aves (Tabla 2).

Tabla 2. Criterios de valoración y puntajes para la variable Período de actividad.

\begin{tabular}{lc}
\hline \multicolumn{1}{c}{ Criterio de valoración } & Puntaje \\
\hline Especies nocturnas, activas cuando no hay luz solar & 0 \\
Especies crepusculares, que desarrollan actividades durante el ocaso & 1 \\
Especies diurnas, con actividades durante el período de luz solar & 2 \\
Especies diurnas-crepusculares, con actividad tanto de día como al ocaso & 3 \\
\hline
\end{tabular}

- Colorido y mimetismo $(\mathbf{C m})$ : grado en que las aves destacan y llaman la atención del observador por sus características cromáticas de plumaje, pudiendo variar desde un mimetismo absoluto con el medio circundante, hasta los casos en que las especies exhiben tonalidades que destacan del entorno y las hacen muy distinguibles (Tabla 3).

Tabla 3. Criterios de valoración y puntajes para la variable Colorido y mimetismo.

\section{Criterio de valoración}

Colorido muy bajo: el color no es una característica destacada, el contraste con el entorno es mínimo o casi inexistente. Plumaje muy mimético.

Colorido medio: el colorido del plumaje puede ser llamativo, pero el contraste con el medio es muy bajo. Son especies con plumajes predominantemente grises, verdosos, pardos, cafés y otros opacos.

Colorido alto: Contraste fuerte con el entorno, además posee colores llamativos para el observador. Predominan el blanco o negro puros.

Colorido muy alto: el colorido exhibe gran contraste con el medio y además posee colores que le confieren una perceptibilidad muy alta. Con tonalidades brillantes y colores fuertes (rojos, amarillos y azules).
Puntajes 
- Tamaño (T): dimensión corporal de los ejemplares observados, que condiciona fuertemente su perceptibilidad. Se asume que mientras más grande sea la especie será más perceptible. El tamaño se documentó con base en Ridgely \& Greenfield (2006) (Tabla 4).

Tabla 4. Criterios de valoración y puntajes para la variable Tamaño

\begin{tabular}{lc}
\hline \multicolumn{1}{c}{ Criterio de valoración } & Puntaje \\
\hline Pequeño: menores de $20 \mathrm{~cm}$ de longitud & 0 \\
Medio: longitud $20-35 \mathrm{~cm}$ & 1 \\
Grande: longitud $35-60 \mathrm{~cm}$ & 2 \\
Muy grande: mayores de $60 \mathrm{~cm}$ de longitud & 3 \\
\hline
\end{tabular}

- $\quad$ Perceptibilidad acústica (Pac): posibilidad de registrar auditivamente la presencia de la especie, por sus llamados y/o cantos. Los llamados son emisiones cortas de voz, mientras que los cantos son series largas de notas armoniosas como una melodía (Tabla 5).

Tabla 5. Criterios de valoración y puntajes para la variable Perceptibilidad acústica.

\begin{tabular}{lc}
\hline \multicolumn{1}{c}{ Criterio de valoración } & Puntajes \\
\hline Sin perceptibilidad acústica: aves que no emiten sonidos perceptibles o con cantos de intensidad reducida. & 0 \\
$\begin{array}{l}\text { Perceptibilidad acústica media: aves que eventualmente emiten cantos, vocalizaciones y éstos son me- } \\
\text { dianamente percibidos. }\end{array}$ & 1 \\
$\begin{array}{l}\text { Perceptibilidad acústica alta: con cantos o vocalizaciones claramente perceptibles, pero no fácilmente } \\
\text { atribuibles a una especie en particular. }\end{array}$ & 2 \\
$\begin{array}{l}\text { Perceptibilidad acústica muy alta: emiten sonidos de altas intensidades, peculiares y atribuibles clara- } \\
\text { mente a una especie en particular. }\end{array}$ & 3 \\
\hline
\end{tabular}

- $\quad$ Conducta (C): ciertos patrones comportamentales llamativos para el observador. Incluyen conductas tales como rituales de apareamiento, formas de vuelo, estilos de alimentación, etc., que pasan a conformar un componente importante en el grado de perceptibilidad de las especies (Tabla 6).

Tabla 6. Criterios de valoración y puntajes para la variable Conducta.

\begin{tabular}{lc}
\hline \multicolumn{1}{c}{ Criterio de valoración } & Puntaje \\
\hline Conducta no llamativa de baja percepción, carece de conductas peculiares. & 0 \\
$\begin{array}{l}\text { Medianamente llamativa, despliegan conductas peculiares, pero temporalmente irregulares o en cier- } \\
\text { tas épocas del año (migraciones, apareamiento). }\end{array}$ & 1 \\
Conducta llamativa de alta perceptibilidad, pero no atribuible a una especie en particular. & 2 \\
$\begin{array}{l}\text { Conducta muy llamativa, además de ser claramente percibida por el observador, conductas atribui- } \\
\text { bles a una especie en particular (estilos de vuelo, conductas agresivas) }\end{array}$ & 3 \\
\hline
\end{tabular}


- $\quad$ Perceptibilidad de indicios (Pi): son evidencias indirectas dejadas por las especies como rastros/ señales (plumas, huellas, nidos). Siendo unos más frecuentes y perceptibles que otras (Tabla 7).

Tabla 7. Criterios de valoración y puntajes para la variable Perceptibilidad de indicios

\begin{tabular}{lc}
\hline \multicolumn{1}{c}{ Criterio de valoración } & Puntaje \\
\hline Sin indicios: aves que no dejan indicios perceptibles o rastros de su presencia. & 0 \\
Perceptibilidad baja: los indicios de estas especies son escasamente perceptibles. & 1 \\
Perceptibilidad media: dejan indicios medianamente perceptibles de sus actividades. & 2 \\
Perceptibilidad alta: dejan indicios muy evidentes de sus actividades (fecas, plumas, huellas, comederos, nidos). & 3 \\
\hline
\end{tabular}

- Grado de tolerancia (Gt): entendida como la servador a un(os) ejemplar(es) de una especie distancia (m) a la que puede acercarse un obdeterminada (Tabla 8).

Tabla 8. Criterios de valoración y puntajes para la variable Grado de tolerancia

\begin{tabular}{lc}
\hline \multicolumn{1}{c}{ Criterio de valoración } & Puntaje \\
\hline Bajo: especies conductualmente tímidas, cautelosas y huidizas del ser humano, muy poco tolerantes. & 0 \\
Medio: cierto nivel de tolerancia a humanos según la estacionalidad y las condiciones del medio. & 1 \\
$\begin{array}{l}\text { Alto: especies que son capaces de tolerar la presencia de seres humanos y no se alejan ante posibles } \\
\text { observadores, pudiendo estos incluso acercarse a unos cuantos metros de distancia. }\end{array}$ & 2 \\
$\begin{array}{l}\text { Muy alto: especies que toleran un grado máximo de acercamiento con humanos, siendo posible } \\
\text { incluso tocarlas. }\end{array}$ & 3 \\
\hline
\end{tabular}

- Estado de Conservación (Ec): estado definido de acuerdo con las categorías según IUCN (2018) (Tabla 9).

Tabla 9. Criterios de valoración y puntajes para la variable Estado de conservación.

\begin{tabular}{cc}
\hline Criterio de valoración & Puntaje \\
\hline Datos insuficientes (DD) y preocupación menor (LC) & 0 \\
Casi amenazadas (NT) & 1 \\
Vulnerables (VU) & 2 \\
En peligro crítico (CR) y en peligro (EN) & 3 \\
\hline
\end{tabular}

- Endemismo (End): nivel de exclusividad de las especies respecto de las distintas unidades territoriales que habitan, desde especies distribuidas únicamente en el área de estudio hasta especies cosmopolitas. La condición de endemismo de las especies fue consultada a partir de Remsen et al. (2018) (Tabla 10). 
Tabla 10. Criterios de valoración y puntajes para la variable Endemismo

\begin{tabular}{lc}
\multicolumn{1}{c}{ Criterio de valoración } & Puntaje \\
\hline Especie no endémica del país & 0 \\
Especie endémica del país & 1 \\
Especie endémica de la región ecológica & 2 \\
Especie endémica del área de estudio y/o área de influencia & 3 \\
\hline
\end{tabular}

- Valor de uso e importancia científica (Vu): con los subparámetros y puntajes de la Tabla 11.

Tabla 11. Criterios de valoración y puntajes para la variable Valor de uso e importancia científica.

\begin{tabular}{lc}
\hline \multicolumn{1}{c}{ Criterio de valoración } & Puntaje \\
\hline $\begin{array}{l}\text { Bajo: especies que no poseen importancia científica o económica documentada. O en su defecto, son espe- } \\
\text { cies muy generalistas. }\end{array}$ & 0 \\
Medio: especies que presentan importancia científica eventual y son capturadas ocasionalmente (caza deportiva). & 1 \\
Alto: Especies con beneficio para el ámbito rural (cinegético u otros) y/o que son de importancia relativa en \\
la estructura de los ecosistemas. En general, su captura está prohibida o regulada. \\
$\begin{array}{l}\text { Muy alto: especies con un alto beneficio en los agroecosistemas y/o de importancia en la estructura de } \\
\text { los ecosistemas (controladores biológicos), cuya cacería además está prohibida. Específicas de bosque } \\
\text { y especialistas. }\end{array}$ \\
\hline
\end{tabular}

- Valor histórico-cultural (Vh): con los subparámetros y puntajes de la Tabla 12.

Tabla 12. Criterios de valoración y puntajes para la variable Valor histórico-cultural

\section{Criterio de valoración}

Puntaje

Muy bajo: especies sin importancia cultural o histórica conocida para el país.

Bajo: especies con baja influencia en aspectos culturales, religiosos, simbólicos u otros de los pueblos originarios, pero que los han acompañado, y por lo mismo son reconocibles.

Medio: especies con un grado considerable de importancia para ciertos aspectos culturales propios de los habi-

- Singularidad taxonómica: nivel de exclusividad o generalidad que presentan las especies en relación con sus niveles de clasificación taxonómica. Si una especie proviene de una línea genética única su valor de singularidad taxonó- mica será más alto. Esta característica refleja el valor del patrimonio genético de la biodiversidad. La singularidad taxonómica se determinó a partir de Data Zone de BirdLife International (2019) (Tabla 13). 
Tabla 13. Criterios de valoración y puntajes para la variable Singularidad taxonómica

\begin{tabular}{lc}
\multicolumn{1}{c}{ Criterio de valoración } & Puntaje \\
\hline Género con cinco o más especies & 0 \\
Género con 2-4 especies & 1 \\
Monotípica al género & 2 \\
Monotípica a la familia o nivel superior & 3 \\
\hline
\end{tabular}

\section{Resultados}

\subsection{Riqueza de especies}

Luego de efectuar 11 transectos, desde septiembre de 2017 hasta marzo de 2018, se registraron 58 especies de aves pertenecientes a 21 familias y 9 órdenes. La familia con mayor número de especies fue Trochilidae (colibríes), con 9. El orden más diverso fue el de los Passeriformes, con 38 especies $(65,5 \%)$ (Tabla 14).

Tabla 14. Riqueza de aves registradas en el sendero a la cascada Cóndor Machay

\begin{tabular}{|c|c|c|c|c|}
\hline $\mathbf{N}^{\mathbf{o}}$ & Nombre común & Identidad taxonómica & Familia & Orden \\
\hline 1 & Pava andina & Penelope montagnii & Cracidae & Galliformes \\
\hline 2 & Pato torrentero & Merganetta armata & Anatidae & Anseriformes \\
\hline 3 & Gaviota andina & Chroicocephalus serranus & Laridae & Charadriformes \\
\hline 4 & Gallinazo negro & Coragyps atratus & Cathartidae & Cathartiformes \\
\hline 5 & Gavilán variable & Geranoaetus polyosoma & Accipitridae & Accipitriformes \\
\hline 6 & Caracara curiquingue & Phalcoboenus carunculatus & Falconidae & Accipitriformes \\
\hline 7 & Cernícalo americano & Falco sparverius & Falconidae & Accipitriformes \\
\hline 8 & Halcón peregrino & Falco peregrinus & Falconidae & Accipitriformes \\
\hline 9 & Tórtola orejuda & Zenaida auriculata & Columbidae & Columbiformes \\
\hline 10 & Paloma collareja & Patagioenas fasciata & Columbidae & Columbiformes \\
\hline 11 & Orejivioleta ventriazul & Colibri coruscans & Trochilidae & Apodiformes \\
\hline 12 & Colacintillo coliverde & Lesbia nuna & Trochilidae & Apodiformes \\
\hline 13 & Colacintillo colinegro & Lesbia victoriae & Trochilidae & Apodiformes \\
\hline 14 & Metalura tiria & Metallura tyrianthina & Trochilidae & Apodiformes \\
\hline 15 & Metalura verde & Metallura williami primolinus & Trochilidae & Apodiformes \\
\hline 16 & Colibrí terciopelo & Lafresnaya lafresnayi & Trochilidae & Apodiformes \\
\hline 17 & Zamarrito colilargo & Eriocnemis luciani & Trochilidae & Apodiformes \\
\hline 18 & Rayito brillante & Aglaeactis cupripenis & Trochilidae & Apodiformes \\
\hline 19 & Colibrí pico de espada & Ensifera ensifera & Trochilidae & Apodiformes \\
\hline 20 & Carpintero dorsicarmesí & Colaptes rivolii & Picidae & Piciformes \\
\hline
\end{tabular}




\begin{tabular}{|c|c|c|c|c|}
\hline 21 & Trepatroncos pardo & Dendrocincla fuliginosa & Furnariidae & Passeriformes \\
\hline 22 & Trepatroncos perlado & Margarornis squamiger & Furnariidae & Passeriformes \\
\hline 23 & Barbablanca rayada & Pseudocolaptes boissonneautii & Furnariidae & Passeriformes \\
\hline 24 & Colaespina de azara & Synallaxis azarae & Furnariidae & Passeriformes \\
\hline 25 & Colaespina rufa & Synallaxis unirufa & Furnariidae & Passeriformes \\
\hline 26 & Gralaria coronicastaña & Grallaria ruficapilla & Grallaridae & Passeriformes \\
\hline 27 & Tapaculo negruzco & Scytalopus latrans & Rhynocriptidae & Passeriformes \\
\hline 28 & Elenia crestiblanca & Elaenia albiceps & Tyrannidae & Passeriformes \\
\hline 29 & Piojito alifranjeado & Mecocerculus stictopterus & Tyrannidae & Passeriformes \\
\hline 30 & Atrapamoscas canelo & Pyrrhomyias cinnamomeus & Tyrannidae & Passeriformes \\
\hline 31 & Febe guardarríos & Sayornis nigricans & Tyrannidae & Passeriformes \\
\hline 32 & Alinaranja golilistada & Myiotheretes striaticollis & Tyrannidae & Passeriformes \\
\hline 33 & Pitajo pechirufo & Ochthoeca rufipectoralis & Tyrannidae & Passeriformes \\
\hline 34 & Pitajo dorsipardo & Ochthoeca fumicolor & Tyrannidae & Passeriformes \\
\hline 35 & Cotinga crestirroja & Ampelion rubrocristatus & Cotingidae & Passeriformes \\
\hline 36 & Golondrina azuliblanca & Pygochelidon cyanoleuca & Hirundinidae & Passeriformes \\
\hline 37 & Golondrina ventricafé & Orochelidon murina & Hirundinidae & Passeriformes \\
\hline 38 & Soterrey colillano & Pheugopedius euophrys & Troglodytidae & Passeriformes \\
\hline 39 & Cinclo gorriblanco & Cinclus leucocephalus & Cinclidae & Passeriformes \\
\hline 40 & Zorzal de Swainson & Catharus ustulatus & Turdidae & Passeriformes \\
\hline 41 & Mirlo grande & Turdus fuscater & Turdidae & Passeriformes \\
\hline 42 & Mirlo negribrilloso & Turdus serranus & Turdidae & Passeriformes \\
\hline 43 & Candelita de anteojos & Myoborus melanocephalus & Parulidae & Passeriformes \\
\hline 44 & Reinita crestinegra & Myothlypis nigrocristata & Parulidae & Passeriformes \\
\hline 45 & Reinita coronirrojiza & Myothlypis coronata & Parulidae & Passeriformes \\
\hline 46 & Reinita plañidera & Geothlypis philadelphia & Parulidae & Passeriformes \\
\hline 47 & Hemispingo superciliado & Hemispingus superciliaris & Thraupidae & Passeriformes \\
\hline 48 & Tangara montana encapuchada & Butrauphis montana & Thraupidae & Passeriformes \\
\hline 49 & Tangara montana ventriescarlata & Anisognathus igniventris & Thraupidae & Passeriformes \\
\hline 50 & Tangara montana pechianteada & Dubusia teniata & Thraupidae & Passeriformes \\
\hline 51 & Tangara azulinegra & Tangara vasorii & Thraupidae & Passeriformes \\
\hline 52 & Picocono cinereo & Conirostrum cinereum & Thraupidae & Passeriformes \\
\hline 53 & Picocono dorsiazul & Conirostrum sitticolor & Thraupidae & Passeriformes \\
\hline 54 & Pinchaflor negro & Diglossa humeralis & Thraupidae & Passeriformes \\
\hline 55 & Pinchaflor enmascarado & Diglossa cyanea & Thraupidae & Passeriformes \\
\hline 56 & Chingolo & Zonotrichia capensis & Passerellidae & Passeriformes \\
\hline 57 & Matorralero cejigris & Arremon assimilis & Passerellidae & Passeriformes \\
\hline 58 & Matorralero nuquirrufo & Atlapetes latinuchus & Passerellidae & Passeriformes \\
\hline
\end{tabular}


Chao 1 estima una cifra de 60,1 especies esperadas, por lo que la curva de acumulación de especies no alcanzó completamente la asíntota. El por- centaje de completitud alcanzado en el muestreo fue de un $96,50 \%$, por lo que es posible afirmar que el muestreo fue representativo (Figura 2).

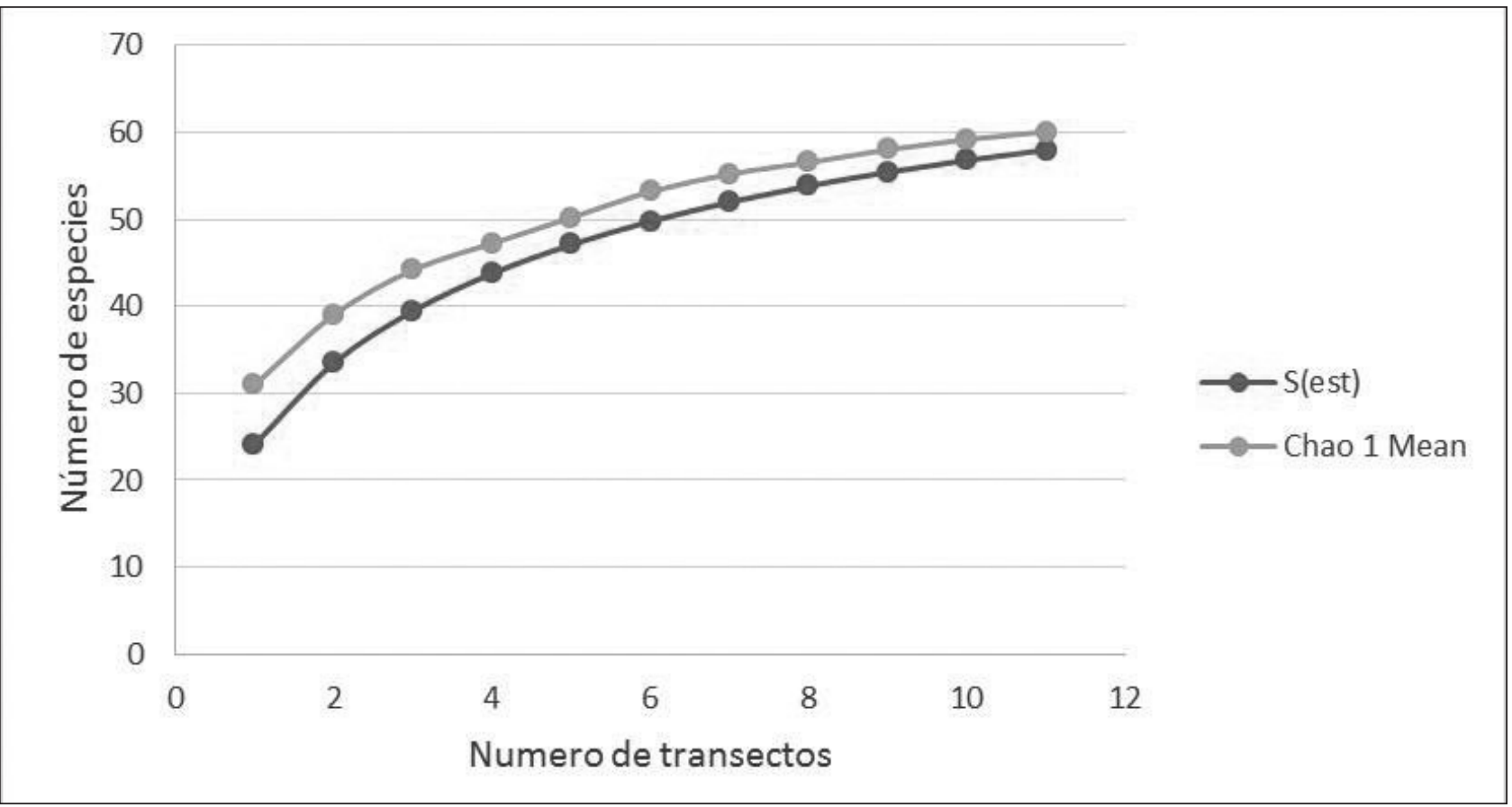

Figura 2. Curva de acumulación y curva de riqueza potencial existente en el área de estudio.

\subsection{Abundancia de especies}

Se registró un total de 1.234 aves. En la Tabla 15 se presentan las cifras de abundancia absoluta de las especies registradas, de mayor a menor. Las cinco especies más abundantes fueron: mirlo grande, tangara montana ventriescarlata, gallinazo negro, piojito alifranjeado y candelita de anteojos. Así mismo, se registraron siete especies que podrían catalogarse como raras para la zona al registrar un único individuo durante todo el muestreo: gavilán variable, colibrí metalura verde, trepatroncos pardo, barbablanca rayada, febe guardarríos, atrapamoscas canelo y el zorzal de Swainson.

Tabla 15. Abundancia absoluta de las aves registradas en el área de estudio

\begin{tabular}{|l|c|c|c|}
\hline \multicolumn{1}{|c|}{ Nombre común } & Total & Nombre común & Total \\
\hline Mirlo grande & 189 & Chingolo & 5 \\
\hline Tangara montana ventriescarlata & 116 & Matorralero cejigris & 5 \\
\hline Gallinazo negro & 79 & Cernícalo americano & 4 \\
\hline Piojito alifranjeado & 71 & Tórtola orejuda & 4 \\
\hline Candelita de anteojos & 68 & Metalura tiria & 4 \\
\hline Caracara curiquingue & 66 & & 4 \\
\hline
\end{tabular}




\begin{tabular}{|c|c|c|c|}
\hline Matorralero nuquirrufo & 64 & Carpintero dorsicarmesí & 4 \\
\hline Golondrina ventricafé & 59 & Elenia crestiblanca & 4 \\
\hline Orejivioleta ventriazul & 46 & Pitajo pechirufo & 4 \\
\hline Tangara azulinegra & 45 & Reinita coronirrojiza & 4 \\
\hline Cinclo gorriblanco & 43 & Halcón peregrino & 3 \\
\hline Trepatroncos perlado & 40 & Tapaculo negruzco & 3 \\
\hline Pava andina & 33 & Tangara montana pechianteada & 3 \\
\hline Paloma collareja & 31 & Rayito brillante & 2 \\
\hline Gralaria coronicastaña & 26 & Colaespina de azara & 2 \\
\hline Pinchaflor enmascarado & 26 & Colaespina rufa & 2 \\
\hline Soterrey colillano & 24 & Pitajo dorsipardo & 2 \\
\hline Mirlo negribrilloso & 23 & Cotinga crestirroja & 2 \\
\hline Picocono cinereo & 19 & Tangara montana encapuchada & 2 \\
\hline Golondrina azuliblanca & 14 & Picocono dorsiazul & 2 \\
\hline Reinita crestinegra & 14 & Pinchaflor negro & 2 \\
\hline Hemispingo superciliado & 11 & Reinita plañidera & 2 \\
\hline Colacintillo colinegro & 10 & Gavilán variable & 1 \\
\hline Alinaranja golilistada & 10 & Metalura verde & 1 \\
\hline Colibrí pico de espada & 8 & Trepatroncos pardo & 1 \\
\hline Gaviota andina & 7 & Barbablanca rayada & 1 \\
\hline Pato torrentero & 5 & Febe guardarríos & 1 \\
\hline Colibrí terciopelo & 5 & Atrapamoscas canelo & 1 \\
\hline Colacintillo coliverde & 5 & Zorzal de Swainson & 1 \\
\hline
\end{tabular}

\subsection{Estructura de la comunidad}

En la Figura 3 se presenta la estructura de la comunidad de las aves del sendero organizadas de mayor a menor - abundancia relativa. Las cinco especies más dominantes fueron: mirlo grande, gallinazo negro, tangara montana ventriescarlata, piojito alifranjeado y candelita de anteojos. El pato torrentero, colibrí terciopelo, colacintillo coliverde, zamarrito colilargo, chingolo, tórtola orejuda aparecen como especies frecuentes, mientras que especies que pueden considerarse como raras fueron el gavilán variable, colibrí metalura verde, trepatroncos pardo, barbablanca rayada, febe guardarríos, atrapamoscas canelo y zorzal de Swainson. Por la forma de la curva se aprecia que la comunidad encontrada es bastante equitativa. 


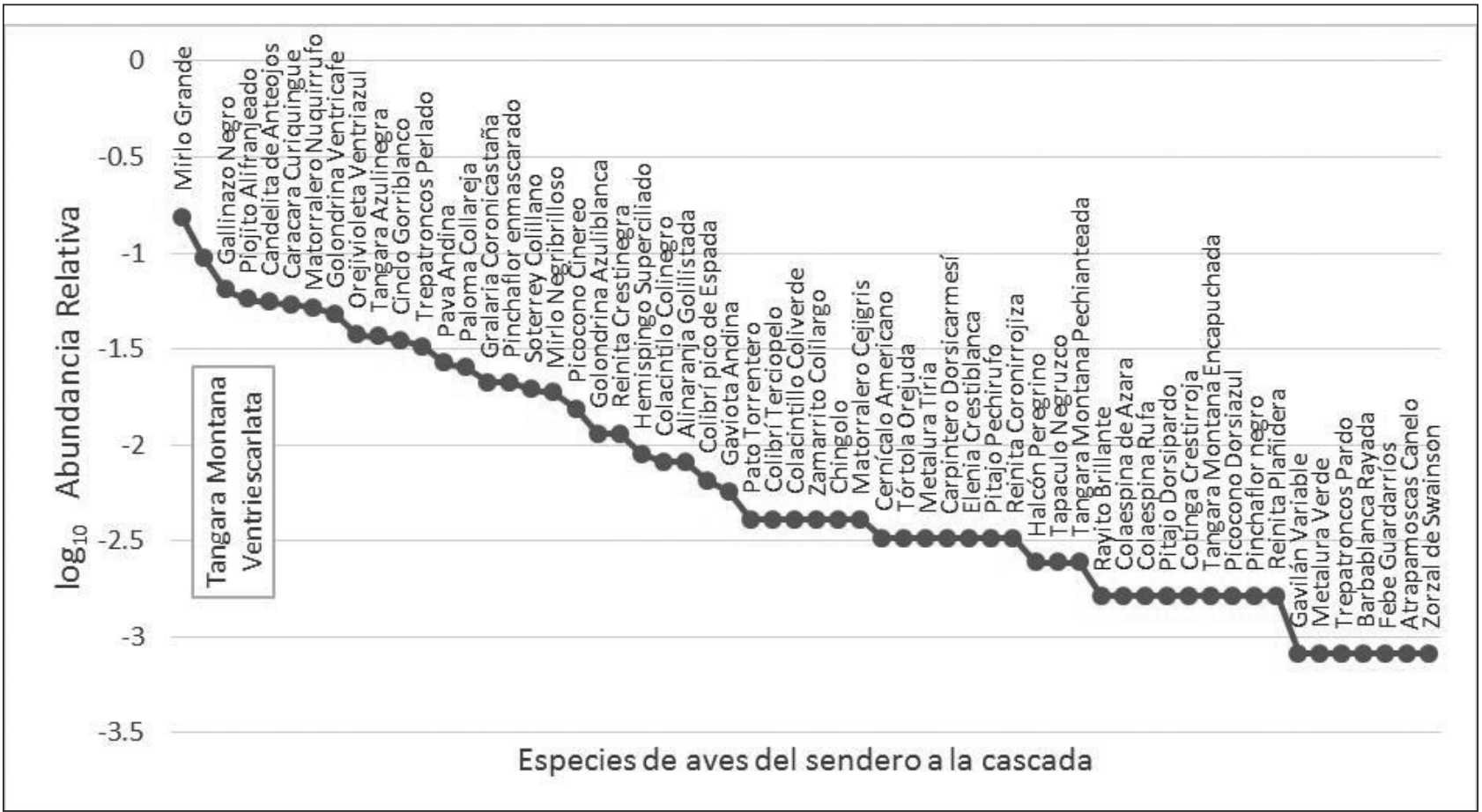

Figura 3. Curva de rango abundancia de la comunidad de aves.

\subsection{Valor estético}

La percepción estética de los 25 entrevistados se incluye en la Figura 4. Se observa que las especies con puntajes superiores a 2,5 son aquellas que presentan plumajes que combinan colores intensos. Dentro de este grupo destacan la familia de las tangaras (Thraupidae) además de algunos colibríes (Trochilidae), un tiránido, el cernícalo americano y la reinita crestinegra. A su vez, especies con los valores más bajos fueron principalmente aquellas con un predominio de colores oscuros en el plumaje, independientemente de su tamaño relativo.

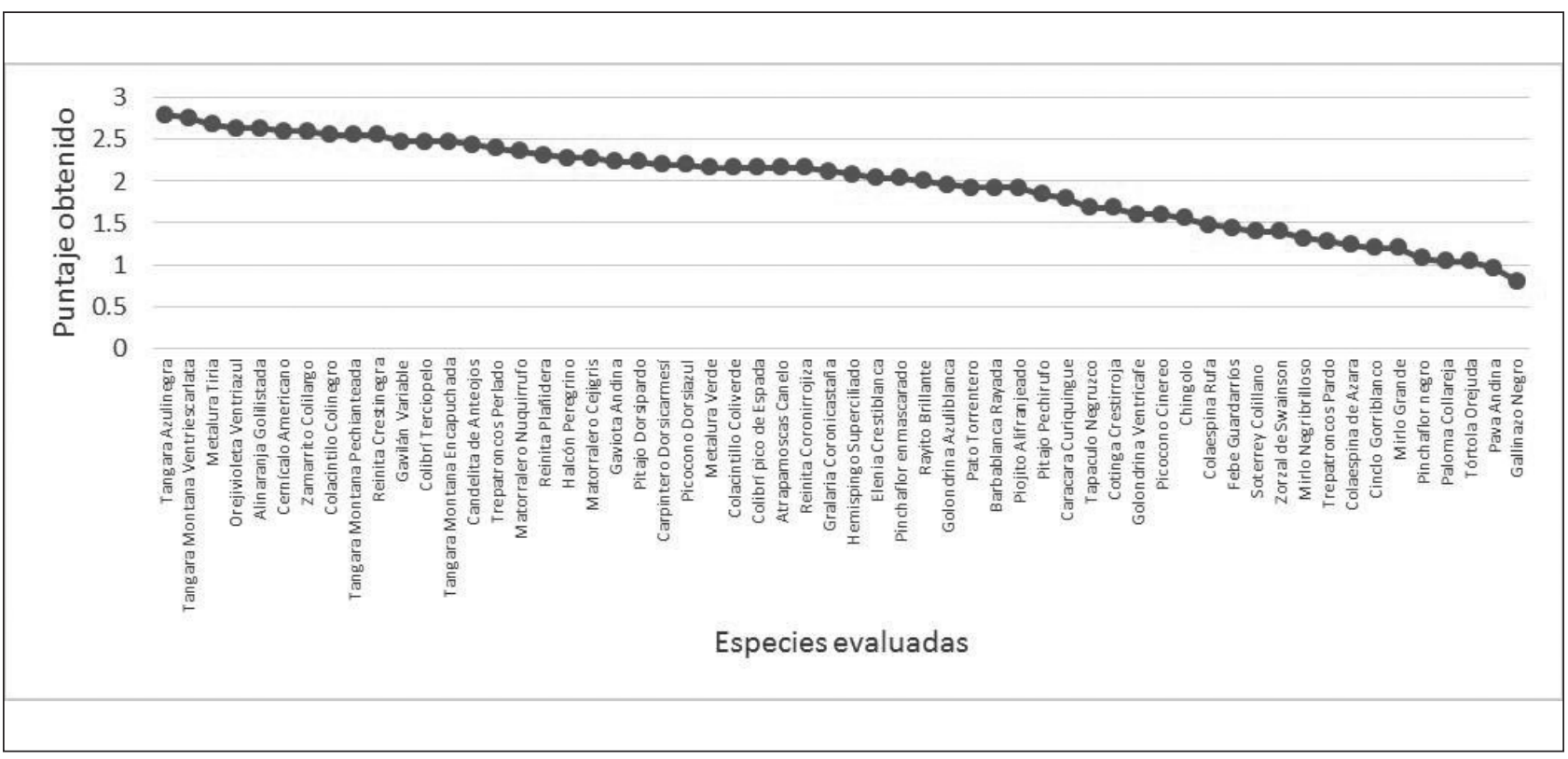

Figura 4. Valor estético de las aves del sendero Cóndor Machay. 


\subsection{Perceptibilidad}

De acuerdo con los datos obtenidos, las especies con mayor valor de perceptibilidad incluyen especies grandes y medianas con plumajes coloridos o especies grandes con plumajes oscuros. En tanto que las especies menos perceptibles son aquellas que tienen tamaño pequeño, que no dejan indicios de su presencia y que usualmente son poco tolerantes ante la presencia humana (Tabla 16).

Tabla 16. Evaluación de la perceptibilidad de las aves del sendero Cóndor Machay

\begin{tabular}{|c|c|c|c|c|c|c|c|c|}
\hline Nombre común & $\mathrm{Pa}^{*}$ & $\mathbf{C m}$ & $\mathbf{T}$ & Pac & $\mathbf{C}$ & $\mathbf{P i}$ & Gt & Perceptibilidad \\
\hline Caracara curiquingue & 3 & 3 & 4,5 & 3 & 3 & 1,5 & 0,5 & 27,75 \\
\hline Gaviota andina & 3 & 3 & 3 & 3 & 3 & 0,5 & 1 & 24,75 \\
\hline Carpintero dorsicarmesí & 4,5 & 4,5 & 1,5 & 2 & 3 & 0,5 & 0 & 24 \\
\hline Tangara Montana ventriescarlata & 4,5 & 4,5 & 1,5 & 3 & 2 & 0 & 0,5 & 24 \\
\hline Mirlo grande & 4,5 & 3 & 1,5 & 2 & 2 & 1 & 1 & 22,5 \\
\hline Pava andina & 3 & 1,5 & 3 & 2 & 3 & 1,5 & 0,5 & 21,75 \\
\hline Cinclo gorriblanco & 3 & 3 & 1,5 & 2 & 3 & 1 & 1 & 21,75 \\
\hline Tangara montana pechianteada & 4,5 & 4,5 & 1,5 & 2 & 2 & 0 & 0 & 21,75 \\
\hline Gallinazo negro & 3 & 3 & 4,5 & 0 & 3 & 0,5 & 0 & 21 \\
\hline Mirlo negribrilloso & 4,5 & 3 & 1,5 & 2 & 2 & 0 & 1 & 21 \\
\hline Matorralero nuquirrufo & 4,5 & 4,5 & 0 & 2 & 2 & 0 & 1 & 21 \\
\hline Candelita de anteojos & 4,5 & 4,5 & 0 & 2 & 2 & 0 & 0,5 & 20,25 \\
\hline Pato torrentero & 3 & 3 & 3 & 1 & 2 & 0,5 & 0,5 & 19,5 \\
\hline Orejivioleta ventriazul & 3 & 4,5 & 0 & 3 & 2 & 0 & 0,5 & 19,5 \\
\hline Colacintillo colinegro & 3 & 4,5 & 1,5 & 1 & 3 & 0 & 0 & 19,5 \\
\hline Colacintillo coliverde & 3 & 4,5 & 1,5 & 1 & 3 & 0 & 0 & 19,5 \\
\hline Colibrí pico de espada & 3 & 4,5 & 1,5 & 1 & 3 & 0 & 0 & 19,5 \\
\hline Tangara montana encapuchada & 4,5 & 4,5 & 0 & 2 & 2 & 0 & 0 & 19,5 \\
\hline Tangara azulinegra & 4,5 & 4,5 & 0 & 2 & 2 & 0 & 0 & 19,5 \\
\hline Alinaranja golilistada & 3 & 4,5 & 1,5 & 2 & 1 & 0 & 0,5 & 18,75 \\
\hline Halcón peregrino & 3 & 3 & 3 & 1 & 2 & 0 & 0 & 18 \\
\hline Hemispingo superciliado & 4,5 & 4,5 & 0 & 2 & 1 & 0 & 0 & 18 \\
\hline Matorralero cejigris & 4,5 & 1,5 & 0 & 2 & 2 & 0 & 1 & 16,5 \\
\hline Reinita crestinegra & 3 & 4,5 & 0 & 1 & 2 & 0 & 0,5 & 16,5 \\
\hline Gavilán variable & 3 & 1,5 & 3 & 1 & 2 & 0 & 0 & 15,75 \\
\hline
\end{tabular}




\begin{tabular}{|c|c|c|c|c|c|c|c|c|}
\hline Rayito brillante & 3 & 4,5 & 0 & 1 & 2 & 0 & 0 & 15,75 \\
\hline Barbablanca rayada & 3 & 3 & 1,5 & 1 & 2 & 0 & 0 & 15,75 \\
\hline Febe guardarríos & 3 & 3 & 0 & 1 & 3 & 0 & 0,5 & 15,75 \\
\hline Pinchaflor enmascarado & 3 & 4,5 & 0 & 1 & 2 & 0 & 0 & 15,75 \\
\hline Reinita coronirrojiza & 3 & 4,5 & 0 & 1 & 2 & 0 & 0 & 15,75 \\
\hline Reinita plañidera & 3 & 4,5 & 0 & 1 & 2 & 0 & 0 & 15,75 \\
\hline Cernícalo americano & 3 & 1,5 & 1,5 & 2 & 2 & 0 & 0 & 15 \\
\hline Tórtola orejuda & 3 & 1,5 & 1,5 & 2 & 2 & 0 & 0 & 15 \\
\hline Gralaria coronicastaña & 3 & 1,5 & 1,5 & 2 & 2 & 0 & 0 & 15 \\
\hline Golondrina azuliblanca & 3 & 3 & 0 & 1 & 2 & 0,5 & 0,5 & 15 \\
\hline Golondrina ventricafé & 3 & 3 & 0 & 1 & 2 & 0,5 & 0,5 & 15 \\
\hline Trepatroncos perlado & 3 & 3 & 0 & 1 & 2 & 0 & 0,5 & 14,25 \\
\hline Pinchaflor negro & 3 & 3 & 0 & 1 & 2 & 0 & 0,5 & 14,25 \\
\hline Paloma collareja & 3 & 1,5 & 1,5 & 1 & 2 & 0 & 0 & 13,5 \\
\hline Piojito alifranjeado & 3 & 1,5 & 0 & 2 & 2 & 0 & 0,5 & 13,5 \\
\hline Cotinga crestirroja & 3 & 1,5 & 1,5 & 2 & 1 & 0 & 0 & 13,5 \\
\hline Soterrey colillano - cucarachero coliliso & 3 & 1,5 & 1,5 & 2 & 0 & 0 & 0 & 12 \\
\hline Picocono cinereo & 4,5 & 1,5 & 0 & 1 & 1 & 0 & 0 & 12 \\
\hline Picocono dorsiazul & 4,5 & 1,5 & 0 & 1 & 1 & 0 & 0 & 12 \\
\hline Metalura tiria & 3 & 1,5 & 0 & 1 & 2 & 0 & 0 & 11,25 \\
\hline Metalura verde & 3 & 1,5 & 0 & 1 & 2 & 0 & 0 & 11,25 \\
\hline Colibrí terciopelo & 3 & 1,5 & 0 & 1 & 2 & 0 & 0 & 11,25 \\
\hline Zamarrito colilargo & 3 & 1,5 & 0 & 1 & 2 & 0 & 0 & 11,25 \\
\hline Elenia crestiblanca & 3 & 1,5 & 0 & 1 & 2 & 0 & 0 & 11,25 \\
\hline Chingolo & 3 & 1,5 & 0 & 1 & 1 & 0 & 1 & 11,25 \\
\hline Colaespina de azara & 3 & 1,5 & 0 & 2 & 0 & 0 & 0 & 9,75 \\
\hline Pitajo dorsipardo & 3 & 1,5 & 0 & 1 & 1 & 0 & 0 & 9,75 \\
\hline Pitajo pechirufo & 3 & 1,5 & 0 & 1 & 1 & 0 & 0 & 9,75 \\
\hline Atrapamoscas canelo & 3 & 1,5 & 0 & 1 & 1 & 0 & 0 & 9,75 \\
\hline Zorzal de Swainson & 3 & 1,5 & 0 & 1 & 1 & 0 & 0 & 9,75 \\
\hline
\end{tabular}




$\begin{array}{lcccccccc}\text { Colaespina rufa } & 3 & 1,5 & 0 & 1 & 0 & 0 & 0 & 8,25 \\ \text { Trepatroncos pardo } & 3 & 1,5 & 0 & 1 & 0 & 0 & 0 & 8,25 \\ \text { Tapaculo negruzco } & 3 & 0 & 0 & 2 & 0 & 0 & 0 & 7,5\end{array}$

*Periodo de actividad (Pa), Colorido y mimetismo (Cm), Tamaño (T), Perceptibilidad acústica (Pac), Conducta (C), Perceptibilidad de indicios (Pi), Grado de tolerancia (Gt).

\subsection{Valor aviturístico potencial (PAV) de las especies evaluadas}

A continuación, se presentan las cifras finales del valor potencial aviturístico de las aves registradas en el área de estudio (Tabla 17).

Tabla 17. Valor aviturístico potencial de las aves del sendero Cóndor Machay

\begin{tabular}{|c|c|c|c|c|c|c|c|c|c|}
\hline Nombre común* & Ve & $\mathbf{A b}$ & Pe & Ec & End & $\mathbf{V u}$ & $\mathbf{V h}$ & St & PAV \\
\hline Caracara curiquingue & 2,7 & 3 & 27,8 & 0 & 0 & 1 & 1,5 & 0 & 35,9 \\
\hline Tangara montana ventriescarlata & 4,14 & 4,5 & 24 & 0 & 0 & 1,5 & 0,5 & 0 & 34,6 \\
\hline Gaviota andina & 3,36 & 1,5 & 24,8 & 0 & 0 & 1,5 & 1 & 0 & 32,1 \\
\hline Mirlo grande & 1,8 & 4,5 & 22,5 & 0 & 0 & 1 & 1 & 0 & 30,8 \\
\hline Carpintero dorsicarmesí & 3,3 & 0 & 24 & 0 & 0 & 1,5 & 1 & 0 & 29,8 \\
\hline Matorralero nuquirrufo & 3,54 & 3 & 21 & 0 & 0 & 1,5 & 0 & 0 & 29,0 \\
\hline Orejivioleta ventriazul & 3,96 & 3 & 19,5 & 0 & 0 & 1,5 & 1 & 0 & 29,0 \\
\hline Candelita de anteojos & 3,66 & 3 & 20,3 & 0 & 0 & 1,5 & 0,5 & 0 & 28,9 \\
\hline Gallinazo negro & 1,2 & 3 & 21 & 0 & 0 & 1,5 & 1 & 1 & 28,7 \\
\hline Pava andina & 1,44 & 3 & 21,8 & 0 & 0 & 1,5 & 1 & 0 & 28,7 \\
\hline Cinclo gorriblanco & 1,8 & 3 & 21,8 & 0 & 0 & 1,5 & 0,5 & 0 & 28,6 \\
\hline Colibrí pico de espada & 3,24 & 1,5 & 19,5 & 0 & 0 & 1,5 & 1 & 1,5 & 28,2 \\
\hline Tangara azulinegra & 4,2 & 3 & 19,5 & 0 & 0 & 1,5 & 0 & 0 & 28,2 \\
\hline Mirlo negribrilloso & 1,98 & 3 & 21 & 0 & 0 & 1 & 1 & 0 & 28,0 \\
\hline Colacintillo colinegro & 3,84 & 1,5 & 19,5 & 0 & 0 & 1,5 & 1 & 0,5 & 27,8 \\
\hline Pato torrentero & 2,88 & 1,5 & 19,5 & 0 & 0 & 1,5 & 1 & 1 & 27,4 \\
\hline Colacintillo coliverde & 3,24 & 1,5 & 19,5 & 0 & 0 & 1,5 & 1 & 0,5 & 27,2 \\
\hline Alinaranja golilistada & 3,96 & 1,5 & 18,8 & 0 & 0 & 1,5 & 1 & 0,5 & 27,2 \\
\hline Tangara Montana pechianteada & 3,84 & 0 & 21,8 & 0 & 0 & 1,5 & 0 & 0 & 27,1 \\
\hline
\end{tabular}




\begin{tabular}{|c|c|c|c|c|c|c|c|c|c|}
\hline Hemispingo superciliado & 3,12 & 3 & 18 & 0 & 0 & 1,5 & 0 & 0 & 25,6 \\
\hline Reinita crestinegra & 3,84 & 3 & 16,5 & 0 & 0 & 1,5 & 0 & 0 & 24,8 \\
\hline Tangara montana encapuchada & 3,72 & 0 & 19,5 & 0 & 0 & 1,5 & 0 & 0 & 24,7 \\
\hline Pinchaflor enmascarado & 3,06 & 3 & 15,8 & 0 & 0 & 1,5 & 0,5 & 0 & 23,8 \\
\hline Halcón peregrino & 3,42 & 0 & 18 & 0 & 0 & 1,5 & 0,5 & 0 & 23,4 \\
\hline Matorralero cejigris & 3,42 & 1,5 & 16,5 & 0 & 0 & 1,5 & 0 & 0 & 22,9 \\
\hline Gralaria coronicastaña & 3,18 & 3 & 15 & 0 & 0 & 1 & 0,5 & 0 & 22,7 \\
\hline Gavilán variable & 3,72 & 0 & 15,8 & 0 & 0 & 1,5 & 1 & 0,5 & 22,5 \\
\hline Golondrina azuliblanca & 2,94 & 3 & 15 & 0 & 0 & 1 & 0 & 0,5 & 22,4 \\
\hline Trepatroncos perlado & 3,6 & 3 & 14,3 & 0 & 0 & 1,5 & 0 & 0 & 22,4 \\
\hline Golondrina ventricafé & 2,4 & 3 & 15 & 0 & 0 & 1 & 0 & 0,5 & 21,9 \\
\hline Rayito brillante & 3 & 0 & 15,8 & 0 & 0 & 1,5 & 1 & 0 & 21,3 \\
\hline Cernícalo americano & 3,9 & 0 & 15 & 0 & 0 & 1 & 1 & 0 & 20,9 \\
\hline Piojito alifranjeado & 2,88 & 3 & 13,5 & 0 & 0 & 1,5 & 0 & 0 & 20,9 \\
\hline Reinita plañidera & 3,48 & 0 & 15,8 & 0 & 0 & 1,5 & 0 & 0 & 20,7 \\
\hline Barbablanca rayada & 2,88 & 0 & 15,8 & 0 & 0 & 1,5 & 0 & 0,5 & 20,6 \\
\hline Paloma collareja & 1,56 & 3 & 13,5 & 0 & 0 & 1,5 & 1 & 0 & 20,6 \\
\hline Reinita coronirrojiza & 3,24 & 0 & 15,8 & 0 & 0 & 1,5 & 0 & 0 & 20,5 \\
\hline Colibrí terciopelo & 3,72 & 1,5 & 11,3 & 0 & 0 & 1,5 & 1 & 1,5 & 20,5 \\
\hline Tórtola orejuda & 1,56 & 1,5 & 15 & 0 & 0 & 1 & 1 & 0 & 20,1 \\
\hline Febe guardarríos & 2,16 & 0 & 15,8 & 0 & 0 & 1 & 0 & 0,5 & 19,4 \\
\hline Zamarrito colilargo & 3,9 & 1,5 & 11,3 & 0 & 0 & 1,5 & 1 & 0 & 19,2 \\
\hline Picocono cinereo & 2,4 & 3 & 12 & 0 & 0 & 1,5 & 0 & 0 & 18,9 \\
\hline Cotinga crestirroja & 2,52 & 0 & 13,5 & 0 & 0 & 1,5 & 0,5 & 0,5 & 18,5 \\
\hline Soterrey colillano & 2,1 & 3 & 12 & 0 & 0 & 1 & 0 & 0 & 18,1 \\
\hline Pinchaflor negro & 1,62 & 0 & 14,3 & 0 & 0 & 1,5 & 0,5 & 0 & 17,9 \\
\hline Metalura tiria & 4,02 & 0 & 11,3 & 0 & 0 & 1,5 & 1 & 0 & 17,8 \\
\hline Metalura verde & 3,24 & 0 & 11,3 & 0 & 0 & 1,5 & 1 & 0 & 17,0 \\
\hline Picocono dorsiazul & 3,3 & 0 & 12 & 0 & 0 & 1,5 & 0 & 0 & 16,8 \\
\hline Chingolo & 2,34 & 1,5 & 11,3 & 0 & 0 & 1 & 0,5 & 0 & 16,6 \\
\hline
\end{tabular}




\begin{tabular}{cccccccccc} 
Elenia crestiblanca & 3,06 & 0 & 11,3 & 0 & 0 & 1,5 & 0 & 0 & $\mathbf{1 5 , 8}$ \\
Atrapamoscas canelo & 3,24 & 0 & 9,75 & 0 & 0 & 1,5 & 0 & 1 & $\mathbf{1 5 , 5}$ \\
Pitajo dorsipardo & 3,36 & 0 & 9,75 & 0 & 0 & 1,5 & 0 & 0 & $\mathbf{1 4 , 6}$ \\
Pitajo pechirufo & 2,76 & 0 & 9,75 & 0 & 0 & 1,5 & 0 & 0 & $\mathbf{1 4 , 0}$ \\
Zorzal de Swainson & 2,1 & 0 & 9,75 & 0 & 0 & 1,5 & 0 & 0 & $\mathbf{1 3 , 4}$ \\
Colaespina de azara & 1,86 & 0 & 9,75 & 0 & 0 & 1 & 0,5 & 0 & $\mathbf{1 3 , 1}$ \\
Trepatroncos pardo & 1,92 & 0 & 8,25 & 0 & 0 & 1,5 & 0 & 0 & $\mathbf{1 1 , 7}$ \\
Colaespina rufa & 2,22 & 0 & 8,25 & 0 & 0 & 1 & 0 & 0 & $\mathbf{1 1 , 5}$ \\
Tapaculo negruzco & 2,52 & 0 & 7,5 & 0 & 0 & 1 & 0 & 0 & $\mathbf{1 1 , 0}$ \\
\hline
\end{tabular}

* Valor estético (Ve), Abundancia relativa (Ab), Perceptibilidad del ave (Pe), Estado de conservación (Ec), Endemismo (End), Valor de uso e importancia científica $(\mathrm{Vu})$, Valor histórico cultural $(\mathrm{Vh})$ y Singularidad taxonómica (St).

Las diez especies con mayor puntaje de valor aviturístico fueron: caracara curiquingue, tangara montana ventriescarlata, gaviota andina, mirlo gran- de, carpintero dorsicarmesí, matorralero nuquirufo, colibrí orejivioleta ventriazul, candelita de anteojos, gallinazo negro y la pava andina (Figura 5).

Figura 5. A) Caracara curiquinge (foto: Javier Torres). B) Tangara montana ventriescarlata. C) Gaviota andina. D) Mirlo grande. E) Carpintero dorsicarmesí. F) Orejivioleta ventriazul. G) Candelita de anteojos (foto: Fernanda Salazar-Vaca). H) Gallinazo negro. I) Pava andina.

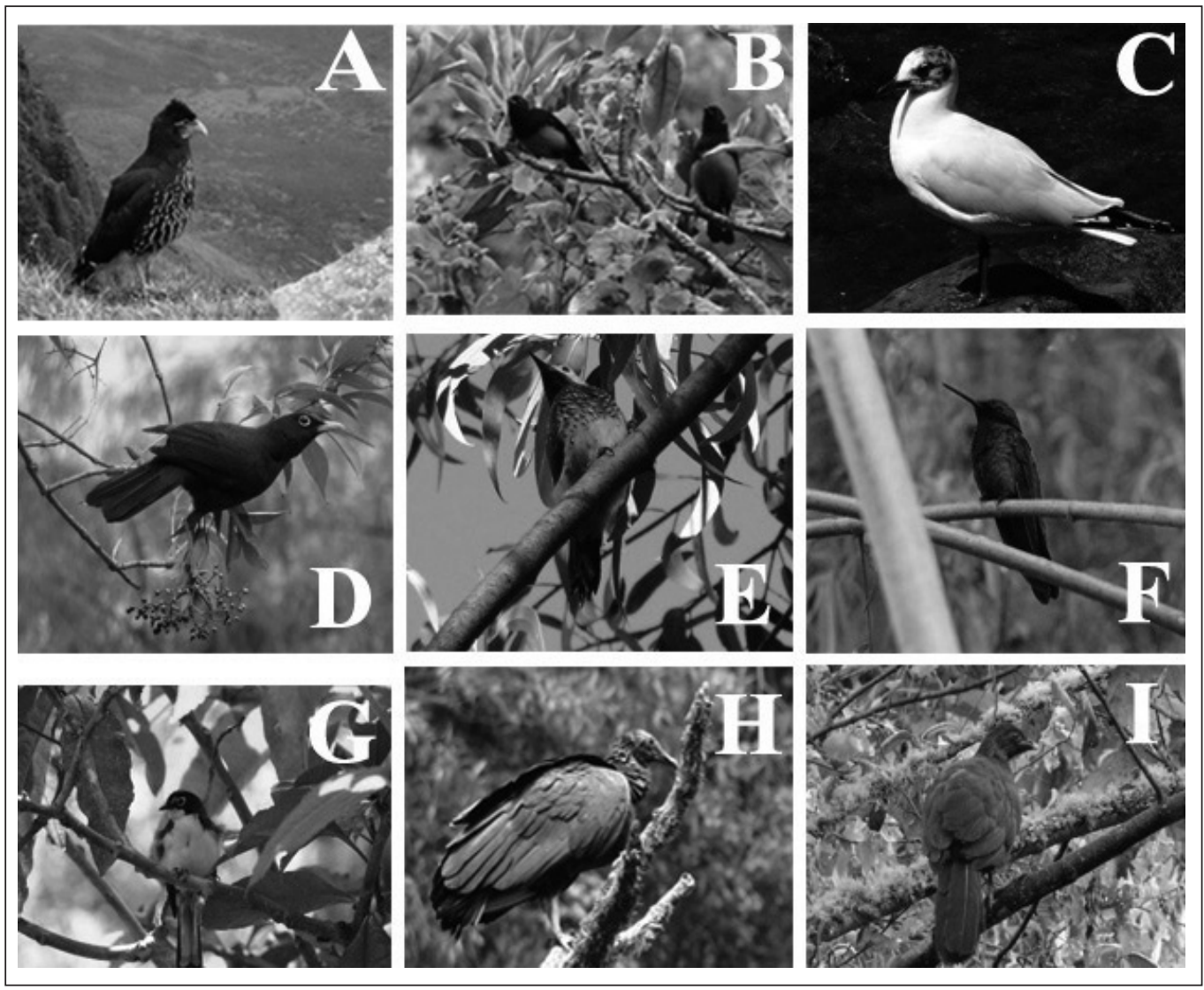




\section{Discusión}

\subsection{Riqueza de especies de aves del sendero}

De acuerdo con Palminteri \& Powell (2001) la riqueza de aves de los bosques montanos de los Andes norte con más de 1.450 especies supera inclusive a la existente en la Amazonía. Su alta diversidad y endemismo se constituyen en características intrínsecas de estas formaciones vegetales. A pesar de ello, el conocimiento acerca de las aves andinas aún es fragmentado, por lo que es importante investigar la diversidad en los remanentes existentes.

Estudios realizados desde un enfoque turístico, reportan cifras comprendidas entre 10 especies (Carmona, 2015) y 41 especies (Herrera, 2016). Herrera (2016) reporta dentro de las 41 especies a la paloma doméstica (Columba livia), colibrí gigante (Patagona gigas) y tiránido (Fluvicola pica). Estas especies, no se han registrado en el presente estudio a pesar del esfuerzo de muestreo. C. livia prefiere hábitats abiertos, cercanos a zonas urbanas, por lo que su mención para el sendero posiblemente sea un error de identificación. El registro de F. pica puede deberse a un error de identificación, considerando que esta especie únicamente presenta registros para la Amazonía ecuatoriana, a alturas entre 350 y 1.900 m s.n.m. (Freile \& Restall, 2018). Probablemente fue confundido con cinclo gorriblanco, especie de plumaje similar, que está presente en el área de estudio. P. gigas no fue registrado, seguramente debido a la pérdida de parches con floripondio Brugmansia sp. que hace algunos años eran más abundantes en la mitad del sendero y que hoy están cubiertas por Chusquea scandens.

Suárez (2013) reporta 24 especies documentadas a partir de revisión bibliográfica y registros de campo. Las especies que difieren a las registradas en este estudio son: chotacabras alifajeado (Caprimulgus longirostris), lechuza blanca (Tyto alba), mochuelo andino (Glaudidium jardinii), mosquero bermellón (Pyrocephalus rubinus), pato de torrente (Anas andium), semillerito coliflajeado (Catamenia analis), cóndor andino (Vultur gryphus), colibrí alizafiro grande (Pterophanes cyanopterus) y al colibrí gigante (P. gigas). En el presente estudio no se logró el registro de aves nocturnas como C. longirostris, T. alba y G. jardinii debido al horario diurno de registro aplicado. La mención de la presencia del mosquero bermellón ( $P$. rubinus) debe ser confirmada, esta especie de tiránido prefiere hábitats más secos, localizados a menor altura en la zona andina (Freile \& Restall, 2018). La referen- cia de $A$. andium como pato de torrente es incorrecta. La cerceta andina (A. andium) prefiere ambientes lacustres, de corriente lenta, que no concuerdan con las características hidrológicas del río Pita. En este estudio, el pato torrentero (M. armata), fue registrado en varias ocasiones dentro del sendero. Finalmente, la cita referida al cóndor andino (Vulthur gryphus) específicamente dentro del sendero debe considerarse únicamente como un registro histórico, considerando que, de acuerdo a los pobladores locales, no se han encontrado cóndores en la zona desde hace 20 años. El único Cathartiforme encontrado actualmente en el sendero fue el gallinazo negro (Coragys atratus).

El sendero en los últimos años también ha sido objeto de estudio como parte de una de las rutas del conteo navideño de aves organizado por el colectivo Aves Quito. En el blog de Aves Quito (2018) se estima la existencia de 60 especies de aves, sin especificar nombres concretos. De esta cifra de riqueza potencial, 58 especies han sido registradas, lo cual es un indicador de una alta variedad para un relicto de bosque montano, cuya superficie es menor a 80 hectáreas. En un recorrido típico realizado desde la siete de la mañana, con un esfuerzo de muestreo de 5 horas, entre 4 personas, se logró el registro de 26 especies de aves cuyas identificaciones están compiladas en la lista S45328595 (eBird, 2018) y no difieren de las especies registradas en este estudio.

Las cifras de riqueza para todo el cantón Rumiñahui varían de 48 (Almeida, 2013) hasta 61 (Lara, 2016). En contraste con la cifra de Lara (2016), el 95 $\%$ de las especies de todo el cantón están presentes en el sendero Cóndor Machay, lo cual amerita promover su conservación a largo plazo. En un estudio de Martínez \& Rochberger (2007) realizado en un bosque andino boliviano de características similares, se logró el registro de 69 especies mediante censos y caminatas, con un esfuerzo de muestreo similar al realizado, pero cubriendo un área de mayor superficie, sin alcanzar la asintonía en la curva de acumulación de especies.

Posiblemente la presencia del río Pita sea un factor que favorece a la riqueza de aves, como sitio de refugio, anidación y alimentación de especies acuáticas, tales como el pato torrentero, gaviota andina y cinclo gorriblanco. Así mismo, la presencia de paredes de roca a ambos lados del río proporciona condiciones favorables para el refugio y la nidificación de las aves rapaces. Finalmente, la composición del relicto de bosque con predominio de especies forestales de la familia Melastomataceae oferta ali- 
mentos para aves frugívoras como la pava andina y las distintas especies de tangaras encontradas.

\subsection{Perspectivas para el aviturismo en el sendero Cóndor Machay}

El aviturismo ha sido notablemente promocionado por el Ecuador, gracias al desarrollo de productos turísticos relacionados a la observación de aves altamente cotizados en el mercado internacional (Espinoza, 2014). Documentos como Actualización de la estrategia nacional de aviturismo (Mindo Cloudforest Foundation, 2010) proporcionan una perspectiva bastante amplia de esta actividad, incluyendo una propuesta de posibles rutas en las cuatro regiones del país, para el conocimiento y uso de posibles aviaficionados y turistas especializados en esta actividad. Sin embargo, se percibe la necesidad de fortalecer la inclusión del turista nacional, tomando en cuenta que muchos de ellos no están especializados para la detección visual de aves pequeñas, poco conspicuas, y tampoco tienen desarrollado un entrenamiento auditivo para reconocimiento de las especies. En este contexto, la promoción del aviturismo a escala nacional debería considerar también estos factores para identificar especies que resulten atractivas para el perfil específico del turista. La inclusión de turistas nacionales en las rutas de aviturismo puede también contribuir a la conservación biológica desde un ámbito local.

Un factor importante para la promoción del aviturismo local es la perceptibilidad de las aves. En estudios realizados en humedales de Argentina y Chile, las aves de mayor tamaño como cisnes, patos y ostreros alcanzaron puntajes más altos (Muñoz-Pedreros \& Quintana, 2010; Almendras et al., 2017); debido a sus períodos diurnos de actividad, plumajes conspicuos, mayores vocalizaciones, conducta llamativa y mayor tolerancia a la presencia humana. En este estudio cuyo enfoque se orientó a las aves del bosque montano, los puntajes más altos fueron para el caracara curiquingue, la gaviota andina y la pava andina como las especies de mayor tamaño corporal registradas, y para otras especies de menor tamaño, pero con plumaje conspicuo, comportamiento distintivo y más vocingleras como el pájaro carpintero dorsicarmesí, tangara montana ventriescarlata, tangara montana pechianteada, cinclo gorriblanco e inclusive el mirlo grande. Para el caso específico de los colibríes, como aves tradicionalmente representativas de los Andes, el colibrí pico de espada (E. ensifera) muy conspicuo por el notable tamaño del pico y los colacintillos (Lesbia sp.) por sus largas colas, fueron las especies con mayores puntajes de perceptibilidad.
En este estudio, los puntajes relacionados con el Estado de conservación (Ec) y Endemismo (End) fueron los más bajos de todas las otras variables que se usan en el cálculo del valor aviturístico potencial (PAV), tomando en cuenta que la estructura de la comunidad de aves estudiada incluyó especies que al momento no están catalogadas bajo categorías de alta amenaza y tampoco son endémicas para el área de estudio. Los valores relativos a Singularidad taxonómica para las aves registradas también fueron bajos para la mayoría de las especies porque se trata de especies que no son monotípicas.

Se recomienda considerar prioritariamente a estas especies para la promoción del aviturismo en el sendero Cóndor Machay. Tomando en cuenta los hallazgos de este estudio, se propone la promoción del sendero de acceso a la cascada Cóndor Machay como un sendero de observación de aves que contribuya al fomento del desarrollo turístico viable y sostenible. Sin embargo, se sugiere considerar las recomendaciones de Vas (2013) para asegurar la sustentabilidad ambiental, socio-cultural y económica del sendero aviturístico con mirada hacia el futuro. En la actualidad se percibe como principal amenaza a la conservación del área y su biodiversidad, la presencia masiva de turistas, especialmente durante los días feriados, que excede notablemente la capacidad de carga efectiva estimada en 268 visitantes por día, conforme recomienda Herrera (2016). Además, los visitantes no cumplen adecuadamente con las normas establecidas acerca del manejo de la basura y la introducción de mascotas dentro del sendero. Otro factor de amenaza para las aves acuáticas es el flujo continuo de excrementos de ganado vacuno que fluyen desde la acequia al canal del río Pita, a mitad del recorrido.

\section{Conclusiones}

Durante la investigación se registró un total de 58 especies de aves, 21 familias y 9 órdenes, siendo un valor elevado en relación con la extensión del área de estudio. Los grupos más diversos fueron colibríes (Trochilidae) y Passeriformes. Las aves con un puntaje más alto del valor aviturístico potencial presentaron un mayor tamaño corporal; además, se incluyen especies de tamaño pequeño, pero con plumaje conspicuo, comportamiento distintivo y cantos fácilmente identificables. Dentro del cálculo, los puntajes relacionados con el Estado de conservación y Endemismo fueron los más bajos de todas las variables usadas. Las especies no están catalogadas bajo categorías graves de amenaza y tampoco son endémicas para el área de estudio. Al concentrar cerca del $95 \%$ de la totalidad de especies 
del cantón Rumiñahui, la actividad aviturística tiene un alto potencial dentro del sendero Cóndor Machay. En la actualidad la principal amenaza para la conservación del área y su biodiversidad es la presencia masiva y poco regulada de turistas, especialmente durante los días feriados. Sin embargo, el desarrollo de proyectos relacionados al aviturismo puede presentarse como alternativas de desarrollo sostenible, si existe un control adecuado de las amenazas identificadas.

\section{Agradecimientos}

El proyecto 6E-2018 Aves del Sendero Cóndor Machay (cantón Rumiñahui, Pichincha, Ecua-

\section{Referencias}

Albuja, L., Almendáriz, A., Barriga, R., Montalvo, D., Cáceres, F., \& Román, J. (2012). Fauna de vertebrados del Ecuador. Quito: Escuela Politécnica Nacional, Instituto de Ciencias Biológicas.

Almeida, P. (2013). Diagnóstico para el diseño de una ruta de turismo de aventura en el cantón Rumiñahui. Pontificia Universidad Católica del Ecuador.

Almendras, A., Ferrari, S., \& Diez, P. (2017). Evaluación de la Avifauna Para Uso Ecoturístico en Humedales del Sur de Santa Cruz. Informes Cientificos Técnicos - UNPA, 9(2), 78-95. https://doi.org/10.22305/ict-unpa.v9i2.247

Amparán, R., \& Téllez, J. (2005). El aviturismo en la zona rural. En Desarrollo rural y turismo (pp. 59-66). Jalisco: Universidad de Guadalajara \& Universidad de Buenos Aires.

Aves Quito. (2019). 10 mejores sitios para observar aves alrededor de Quito. Obtenido de: http:// avesquito.blogspot.com/2018/02/10-mejores-sitios-para-observar-aves.html

BirdLife International. (2019). Data Zone. Obtenido de: http://datazone.birdlife.org/species/ results? thrlev $1=\&$ thrlev $2=\& \mathrm{kw}=\&$ fam $=0 \&$ gen $=0 \& \mathrm{spc}=\& \mathrm{cmn}=\&$ reg $=0 \& \mathrm{cty}=0$

CAF-Banco de Desarrollo de América Latina. (2014). Biocomercio andino: quince historias de éxito dor): Evaluación de especies con valor para el aviturismo local, fue financiado mediante el respaldo institucional y la aprobación del Consejo Directivo de la Facultad de Ciencias Biológicas de la Universidad Central del Ecuador, mediante resolución N. ${ }^{\circ}$ 0106-CD-FCB-2017.

A Lida Guarderas-Flores e Iván Jácome-Guarderas por su contribución durante toda la fase de campo. A Santiago Buitrón y Wladimir Banda por la revisión del Abstract. Muchas gracias también a Javier Torres y Fernanda Salazar-Vaca por su contribución con las fotografías respectivas, y a Paulina Guarderas por la elaboración del mapa del área de estudio.

en Colombia, Ecuador y Perú. Lima: CAF. http://scioteca.caf.com/handle/123456789/518

Cadena-Ortiz, H., Varela, S., Bahamonde-Vinueza, D., Freile, J., \& Bonaccorso, E. 2015. Birds of the Bosque Protector Jerusalem, Guayllabamba Valley, Ecuador. Check List, 11(5), 1-8. http:// dx.doi.org/10.15560/11.5.1770

Carmona, E. (2015). Plan de mejoramiento turístico del sendero Cascada del Pita, en la parroquia de Rumipamba, cantón Rumiñahui. Universidad Tecnológica Israel.

Chandi, A., \& Muñoz, Y. (2018). Aviturismo, alternativa de conservación de la avifauna de la comunidad Gualchán, provincia del Carchi. Tierra Infinita, 4(1), 49-68. https://doi. org/10.32645/26028131.745

Colwell, R. K. (2013). EstimateS: Statistical estimation of species richness and shared species from samples. Version 9. User's Guide and application.

Cuesta, F., Peralvo, M., \& Valarezo, N. (2009). Los bosques montanos de los Andes tropicales. Una evaluación regional de su estado de conservación y de su vulnerabilidad a efectos del cambio climático. Quito: Iniciativa Regional de Estudios Ambientales Andinos, CONDESAN.

eBird. (2018). Lista de aves S45328595. Cascada Cóndor Machay, Pichincha EC., Ob- 
tenido de: https://ebird.org/view/checklist/ S45328595

El-Mahdy, A., \& Abdel-Motaal, D. (2018). Avitourism: Non-Traditional Type of Tour-Guiding: in Egypt. Minia Journal of Tourism and Hospitality Research, 2, 92-104.

Espinoza, F. (2014). Estudio de mercado para el aviturismo en la provincia del Guayas. Guayaquil: Prefectura del Guayas - Guayas Turístico - Biocomercio Andino.

Feinsinger, P. (2003). El diseño de estudios de campo para la conservación de la biodiversidad. Santa Cruz de la Sierra: Editorial FAN.

Fjeldså, J. (2007). The relationship between biodiversity and population centres: the high Andes as an example. Biodiversity and Connservation, 16(10), 2739-2751. https://doi.org/10.1007/ s10531-007-9204-4

Freile, J., \& Rodas, F. (2008). Conservación de aves en Ecuador: ¿cómo estamos y qué necesitamos hacer? Revista Cotinga, 29, 48-55.

Freile, J., \& Restall, R. (2018). Birds of Ecuador. London: Helm Field Guides.

Gobierno Autónomo Descentralizado de Rumiñahui. (2011). Atlas de biodiversidad del cantón Rumiñahui. Sangolquí: GAD Rumiñahui.

Gobierno Autónomo Descentralizado Parroquial de Rumipamba. (2012). Plan de Desarrollo y Ordenamiento Territorial de la Parroquia Rumipamba 2012-2025. Quito: GADP de Rumipamba.

Guarderas, L., \& Jácome, I. (2010). Aves del cantón Rumiñahui. Tomo I. Primera edición. Quito: Gráficas Iberia.

Herrera, Y. (2016). Modelo de gestión turística para la cascada Cóndor Machay, ubicada en la parroquia Rumipamba, cantón Rumiñahui, provincia de Pichincha. Pontificia Universidad Católica del Ecuador.

Herrera, S., \& Lasso, S. (2014). Belleza y colorido de las aves, una experiencia incomparable en Mindo. Revista Kalpana, 12, 6-21.

Hostettler, S. (2002). Tropical montane cloud forest: a challenge for conservation. Bois et forets des Tropiques, 274(4), 19-31.
IUCN - Unión Internacional para la Conservación de la Naturaleza. (2018). The IUCN Red List of Threatened Species. Version 2017.3. Obtenido de: http://www.iucnredlist.org

Josse, C., Cuesta, F., Navarro, G., Barrena, V., Cabrera, E., Chacón-Moreno, E., Ferreira, W., Peralvo, M., Saito, J., \& Tovar, A. (2009). Ecosistemas de los Andes del norte y centro. Bolivia, Colombia, Ecuador, Perú $y$ Venezuela. Lima: Secretaría General de la Comunidad Andina, Programa Regional ECOBONA-Intercooperation, CONDESAN-Proyecto Páramo Andino, Programa BioAndes, EcoCiencia, NatureServe, IAvH, LTA-UNALM, ICAE-ULA, CDC-UNALM, RUMBOL SRL.

Lara, A. (2016). Diseño de un sistema de conservación para el cantón Rumiñahui. Universidad Técnica Particular de Loja.

Llulluna, S. (2018). Estudio de factibilidad para la implementación de un producto de aviturismo en la parroquia Cosanga, provincia de Napo. Escuela Superior Politécnica de Chimborazo.

MAE - Ministerio del Ambiente del Ecuador, \& FAO - Organización de las Naciones Unidas para la Alimentación y la Agricultura. (2015). Especies forestales leñosas arbóreas y arbustivas de los bosques montanos del Ecuador. Quito: MAE \& FAO.

McMullan, M., \& Vásquez, A. (2009). Birds of the Norwest Ecuador. Quito: Spotfield books.

McMullan, M., \& Navarrete, L. (2017). Fieldbook of the Birds of Ecuador, including the Galapagos Islands and common mammals. Quito: Ratty Ediciones.

Martínez, O., \& Rochberger,J. 2007. Características de la avifauna en un gradiente altitudinal de un bosque nublado andino de La Paz, Bolivia. Revista Peruana de Biología, 14(2), 225-236.

Mindo Cloudforest Foundation. (2006). Estrategia Nacional de Aviturismo. Quito: CORPEI.

Muñoz, A. (2015). La contradicción del turismo en la conservación y el desarrollo en Galápagos-Ecuador. Estudios y perspectivas en Turismo, 24, 399-413. 
Muñoz-Pedreros, A., \& Quintana, J. (2010). Evaluación de fauna silvestre para uso ecoturístico en humedales del río Cruces, sitio Ramsar de Chile. Revista Interciencia, 35(10), 730-738.

Muñoz-Pedreros, A. (2004). La evaluación del paisaje: una herramienta de gestión ambiental. Revista Chilena de Historia Natural, 77, 139-156. http://dx.doi.org/10.4067/S0716078X2004000100011

Palminteri, S., \& Powell, G. (2001). Visión de la biodiversidad de los Andes del norte. Colombia: World Wilflife Fund.

Pérez-Sato, J. A., Alcántara-Salinas, G., García-García, C. G., Rivera-Hernández, J. E., Salazar-Ortiz, J., Campos-Cerón, M., Román-Hernández, D., Balderas-San Miguel, O., \& Fuentes-Moreno, A. (2018). Aviturismo en la región de las altas montañas de Veracruz, México. Revista Agroproductividad, 11(8), 101-108. https://doi. org/10.32854/agrop.v11i8.1104

Price, M., Gratzer, G., Alemayehu Duguma, L., Kohler, T., Maselli, D., \& Romeo, R. (editors). (2011). Mountain Forests in a Changing World - Realizing Values, addressing challenges. Rome: FAO/MPS and SDC.

Remsen, J., Areta, J., Cadena, C., Claramunt, S., Jaramillo, A., Pacheco, J. Robbins, M., Stiles, F., Stotz, D., \& Zimmer, K. (2018). A classification of the bird species of South America. American Ornithologists' Union. Versión 2018. Obtenido de: http://www.museum.lsu.edu/ Remsen/SACCBaseline.html

Ridgely, R., \& Greenfield. P. (2006). Aves del Ecuador. Volúmenes 1 y 2. Quito: Academia de Ciencias Naturales de Filadelfia \& Fundación de Conservación Jocotoco.
Steven, R. (2017). Mejorando los beneficios del aviturismo. Ideas y oportunidades. Revista Decisión Point, E03, 7-8.

Sociedad Civil del Cantón Rumiñahui. (2001). Plan estratégico participativo del cantón Rumiñahui 2002-2022. Primera edición. Sangolquí.

Suárez, F. (2013). Propuesta para la implementación de facilidades y planta turística especializada para el desarrollo del proyecto turístico comunitario Cóndor-Machay, cantón Rumiñahui, provincia de Pichincha. Universidad Internacional del Ecuador.

Tejedor, N., Álvarez, E., Arango Caro, S., Araujo Murakami, A., Blundo, C., Boza Espinoza, T., La Torre, M., Gaviria, J., Gutiérrez, n., Jørgensen, P., León, B., López Camacho, R., Malizia, L., Millán, B., Moraes, M., Pacheco, S., Rey Benayas, J., Reynel, C., Timaná de la Flor, M., Ulloa, C., Vacas Cruz, O., \& Newton A.C. (2012). Evaluación del estado de conservación de los bosques montanos en los Andes tropicales. Revista Ecosistemas, 21(1-2), 148-166.

Vas, K. (2013). Birding trails as sustainable tourism development. OIDA International Journal of Sustainable Development, 06(03), 23-34.

Verástegui, I. (2013). El primer "Birding Rally Challenge". Xilema, 26(1), 18-24. http://dx.doi.org/10.21704/x.v26i1.610

Villarreal, H., Álvarez M., Córdova S., Escobar F., Fagua G., Gast F., Mendoza H., Ospina M., \& Umaña A. (2004). Manual de métodos para el desarrollo de inventarios de biodiversidad. Bogotá: Programa de inventarios de biodiversidad. Instituto de Investigación de Recursos Biológicos Alexander von Humboldt. 